\title{
Estimation of the High Resolution Wind Field at Galway Bay
}

\author{
Lei Ren, Jerome Sheahan, Stephen Nash, Diarmuid Nagle, and Michael Hartnett
}

\begin{abstract}
Hydrodynamic circulation in estuaries is primarily driven by tides, surface wind and river inflows. While tidal and river data can be quite easily obtained for input to hydrodynamic models, sourcing accurate surface wind data are problematic. Firstly, the wind data used in hydrodynamic models are usually measured on land and can be quite different in magnitude and direction from real offshore wind. Secondly, surface wind is spatially-varying but due to a lack of data, usually a non-varying wind speed and direction are specified across the full extents of a model domain. These problems can lead to inaccuracies in the surface currents computed by two-dimensional or three-dimensional hydrodynamic models. In the present research, a wind forecast model was used to generate a high resolution wind field at a marine renewable energy test site, Galway Bay. These predicted high resolution wind date can be used to investigate the effect of surface wind data resolution on model accuracy.
\end{abstract}

Index Terms-Wind field, high resolution, Galway bay, ARIMA.

\section{INTRODUCTION}

The directional influence of wind on surface currents is believed to be isotropic in the open ocean in agreement with Ekman theory [1], [2]. The behavior of wind-driven flow in coastal regions, however, is much more complex due to frictional resistance exerted by varying bottom topography and relatively shallow depths [3]. This can result in complex circulation patterns within an estuary or bay and may lead to the formation of gyres. Hydrodynamic circulation in estuaries is primarily driven by tides, river inflows and surface wind. A numerical model will therefore require boundary data for the tidal, river inflows and wind forcing. The accuracy of these data is extremely important for model accuracy. While accurate tidal and river data can be quite easily obtained, sourcing accurate surface wind data are problematic. The use of non-spatially-varying, land-based measurements is quite common due to the unavailability of offshore wind data. However, offshore wind may be substantially stronger than onshore wind and there can be significant spatial variation if wind is over large areas, particularly in coastal areas. The use of non-spatially varying onshore wind data for models can result in the inaccurate prediction of surface currents in

Manuscript received March 9, 2015; revised August 27, 2015. This research was supported by Ryan Institute and the Department of Civil Engineering, National University of Ireland, Galway and China Scholarship Council (CSC). This research was carried out with the support of funding from the MAREN2 and Energy MARE projects which are part-funded by the European Regional Development Fund (ERDF) through the Atlantic Area Transnational Programme (INTERREG IV).

The authors are with the Ryan Institute, National University of Ireland, Galway, Ireland (e-mail: leirencomeon@gmail.com). models. In order to produce the high resolution wind field from modeling, a wind prediction model was developed at a marine renewable energy test site, which is shown in Fig. 1.

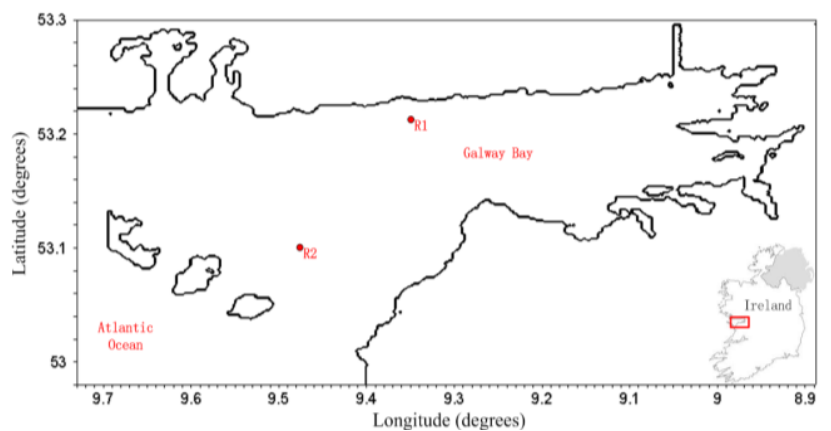

Fig. 1. Research domain (R1 and R2 indicate two random points to extract time series of wind data for comparison).

The paper is outlined as follows. Section II introduces the available wind data in the test site. The wind prediction model is presented in Section III. Section IV presents the results of predicted high resolution wind fields. Conclusions are presented in Section V.

\section{WIND DATA}

Wind data used in this research included measured onshore wind data and unsuccessive forecast offshore wind data. The available wind data were from Julian Day 274 to Julian Day 334,2011 . The first wind data were from the weather station of Informatics Research Unit for Sustainable Engineering (IRUSE) located in the campus of National University of Ireland, Galway, which is approximately $5 \mathrm{~km}$ from the northeastern coast of Galway Bay. Wind data were recorded every minute with speed measured in $\mathrm{m} / \mathrm{s}$ and direction given in degrees from north. The second wind data comprised of a series of short term high resolution forecast of offshore wind during the same period as follows:

1) Period A: Julian Day 291, 2011 18:00 to Julian Day 294, 2011 00:00 (55hrs)

2) Period B: Julian Day 301, 2011 12:00 to Julian Day 302, 2011 17:00 (29hrs)

3) Period C: Julian Day 309, 2011 18:00 to Julian Day 3012, 2011 00:00 (55hrs)

4) Period D: Julian Day 312, 2011 00:00 to Julian Day 314, 2011 06:00 (55hrs)

The second unsuccessive high resolution wind data were obtained from a wind forecast model Hirlam-Aladin Research towards Meso-scale Operational NWP in Europe (HARMONIE), which used a $0.5 \mathrm{~km}$ grid nested within the coarse grid with sixty vertical levels and twelve seconds time step and driven by European Centre for Medium-range 
Weather Forecasting (ECMWF) boundary conditions [4], [5]. The forecast wind data were averaged to produce hourly averaged wind field. Fig. 2 shows one vector map of high resolution wind data in domain.

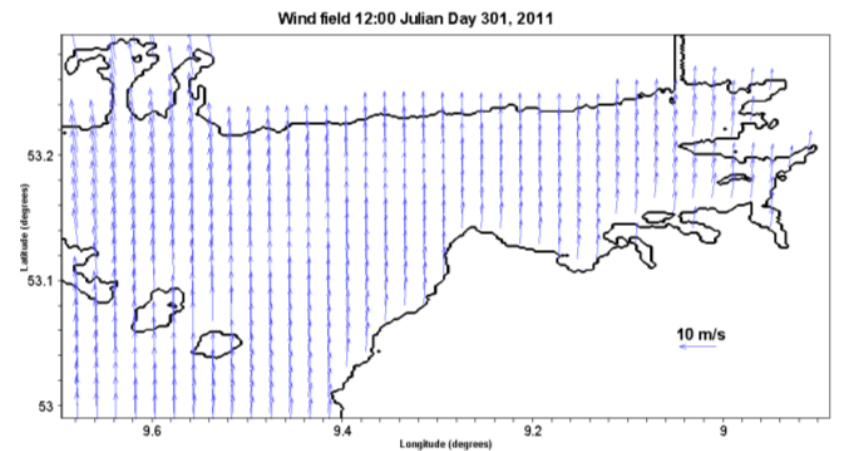

Fig. 2. Vector map of high resolution wind field.

In order to analyze the two wind datasets, time series of wind speed and wind direction are shown in Fig. 3 and Fig. 4. Time series of high resolution were extracted at point R2 (see Fig. 1) in Galway Bay.

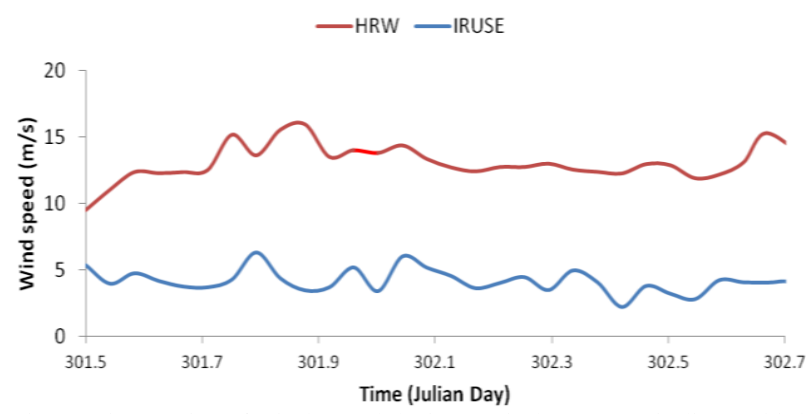

Fig. 3. Time series of wind speed during period B (HRW indicates high resolution wind data; IRUSE indicates wind data from onshore station).

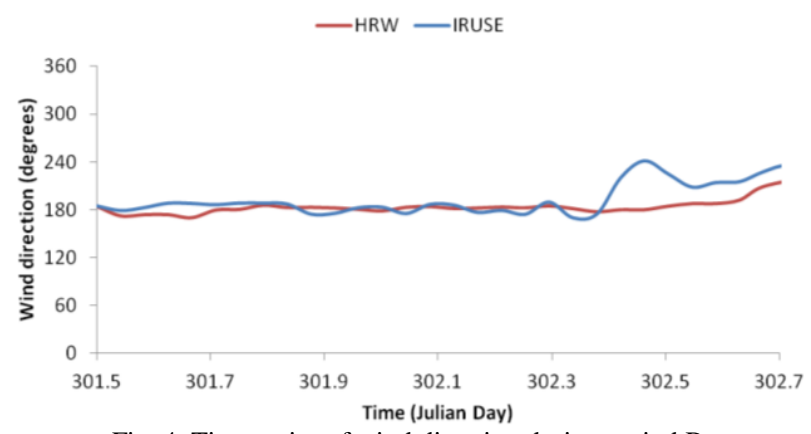

Fig. 4. Time series of wind direction during period B.

Fig. 3 and Fig. 4 show that general direction of the wind data taken from IRUSE weather station matches well with the high resolution wind data; while the wind speed shows significant variation from each other. Wind speed extracted from high resolution wind data was always greater than IRUSE wind speed. This may result from the IRUSE measurement station is located onshore. In order to further compare the two wind datasets, wind roses of IRUSE data and unsuccessive high resolution wind data are shown in Fig. 5 and Fig. 6.

Wind roses at two locations also show that two wind data have similar trend, but the magnitudes are different. In this research, the authors focused on predicting spatially varying wind speed.

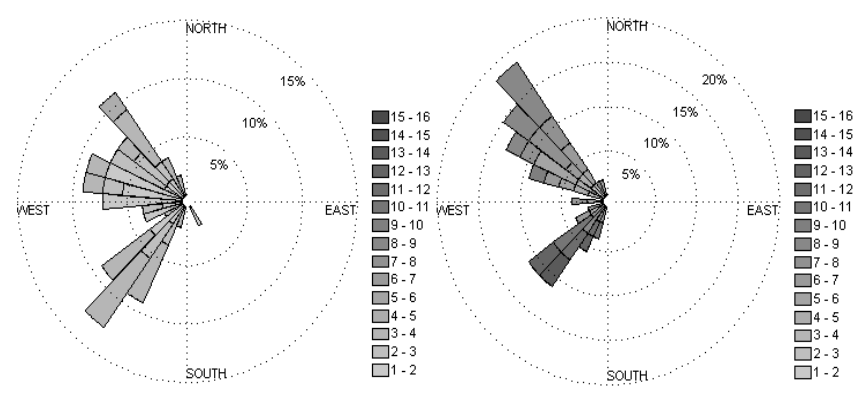

Fig. 5. Wind rose of point R1 in Fig. 1 of period A (Left panel is from IRUSE, right panel is from ARIMA model).

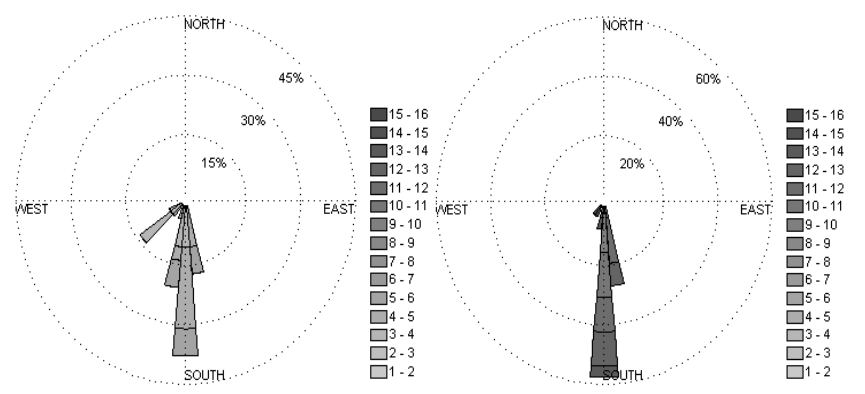

Fig. 6. Wind rose of point R2 in Fig. 1 of period B (Left panel is from IRUSE, right panel is from ARIMA model).

\section{MethodologIES}

In order to produce a continuous temporally and spatially-varying wind dataset, a wind prediction model was developed based on land-measured wind data and limited forecast of high resolution offshore wind from model HARMONIE. Wind data from IRUSE were used for the entire period.

The goal of this work was to forecast the high resolution wind field using Box-Jenkins Autoregressive Integrated Moving Average (ARIMA) modeling based on unsuccessive high resolution wind data and successive IRUSE wind data [6], [7]. A non-seasonal ARIMA model is often donated as $\operatorname{ARIMA}(p, d, q), p$ is the order of the auto-aggressive component and $q$ is the order of the moving average component fitted to the $d$-th differences of the series. The forecasting process can be sequentially listed as below:

Step one: Fit a model to the time series of differences between observed IRUSE wind data and predicted high resolution wind data.

Step two: Fit a model to produce the time series of IRUSE wind data during forecasting period.

Step three: Generate the high resolution wind model based on the difference equation.

In these steps, fitting a model means finding some function of time, which fits the observed values of $D_{t}$ that may be used to forecast future values and /or interpolate missing values. ARIMA model expressed that predicted $D_{t}$ as a function of either previous predicted values of $D_{t}$ at earlier time points (the autoregressive terms) and/ or terms involving random shocks at previous time points (the moving average terms).

Note that a random shock $\varepsilon_{t}$ at time $t$ is the difference between a random observation and its expected value, that is, $\varepsilon_{t}=D_{t}-E\left(D_{t}\right)$. An observed value of a random shock will be denoted as $\varepsilon_{t}^{0}=D_{t}-D_{t}^{0}$. This is also called the residual at time $t$ (the amount by which the observed and predicted 
values differ). The software used to fit the ARIMA model was SPSS (Statistical Package for the Social Sciences), which essentially automates the entire procedure and chooses the best selection of values of $p, d$ and $q$ from among all $\operatorname{ARIMA}(p, d, q)$ models [8]. As it transpired, the best ARIMA model was a simple exponential smoothing model, called a $\operatorname{ARIMA}(0,1,1)$ without constant. The fitted model produced by SPSS is in the form $(0,1,1)$, which can be expressed as [9]:

$$
\begin{gathered}
D_{t}^{p}=D_{t-1}^{p}-0.949 \times \varepsilon_{t-1}^{p} \\
\varepsilon_{t}^{p}=D_{t}^{o}-D_{t}^{p}
\end{gathered}
$$

where,

$D_{t}^{p}$ is the prediction difference at time $t$;

$D_{t}^{o}$ is the observation difference at time $t$;

$\varepsilon_{t}^{p}$ is the residual at time $t$.

The fitted model, equation (1), expressed that at any time $t$, the predicted difference between IRUSE and high resolution wind data is equal to the predicted difference at time $(t-1)$ minus 0.949 times the difference between the observed and the predicted values at time $(t-1)$.

The aim of the fitted model was to predict high resolution wind data speed $W_{t}^{H R P}$ from IRUSE wind data $W_{t}^{\text {IRUSEo }}$. Since observation difference $D_{t}^{o}=W_{t}^{H R p}-W_{t}^{\text {IRUSEo }}$, so high resolution wind data can be obtained based on SPSS in the following formula:

$$
\begin{gathered}
W_{t}^{\text {IRUSE } p}=W_{t-1}^{\text {IRUSE }}-0.943 \times \varepsilon_{t-1}^{\text {IRUSE }} \\
\varepsilon_{t}^{\text {IRUSE }}=W_{t}^{\text {IRUSEo }}-W_{t}^{\text {IRUSE } p}
\end{gathered}
$$

where,

$$
\begin{aligned}
& W_{t}^{I R U S E o} \text { is the observation of IRUSE data at time } t \text {; } \\
& W_{t}^{I R U S E p} \text { is the prediction of IRUSE data at time } t \\
& \varepsilon_{t}^{I R U S E} \text { is the residual of IRUSE data at time } t .
\end{aligned}
$$

In order to forecast high resolution wind data $W_{t}^{H R p}$, a fitted model for high resolution wind data $W_{t}^{H R p}$ can be obtained using $W_{t}^{H R p}=W_{t}^{\text {IRUSEp }}+D_{t}$.

$$
\begin{aligned}
& W_{t}^{H R p}=\left(W_{t-1}^{\text {IRUSEp }}-0.943 \times\left(W_{t-1}^{\text {IRUSEo }}-W_{t-1}^{\text {IRUSEp }}\right)\right) \\
& +\left(D_{t-1}^{p}-0.949 \times\left(D_{t-1}^{o}-D_{t-1}^{p}\right)\right)
\end{aligned}
$$

\section{RESULTS}

Since the advantages of ARIMA wind data is that predicted data are spatially varying compared with IRUSE wind data, contours of wind speed components and vector maps of a predicted wind field from ARIMA model are shown in Fig. 7, Fig. 8, and Fig. 9.

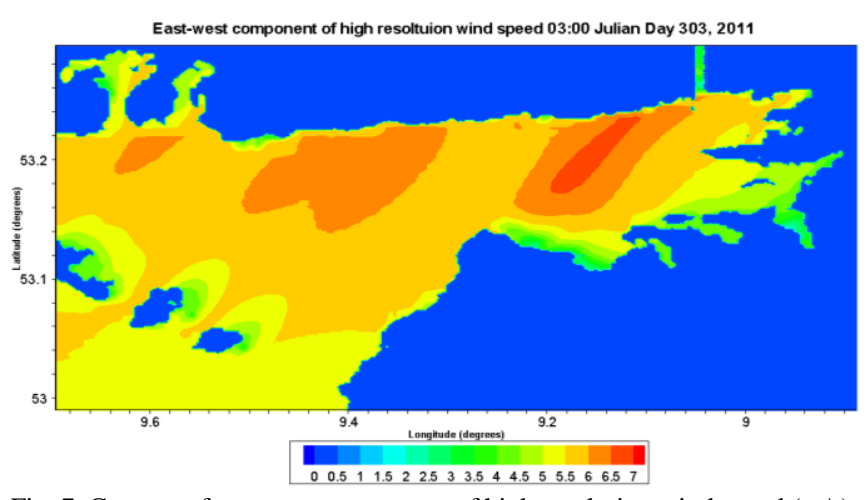

Fig. 7. Contour of east-west component of high resolution wind speed (m/s).

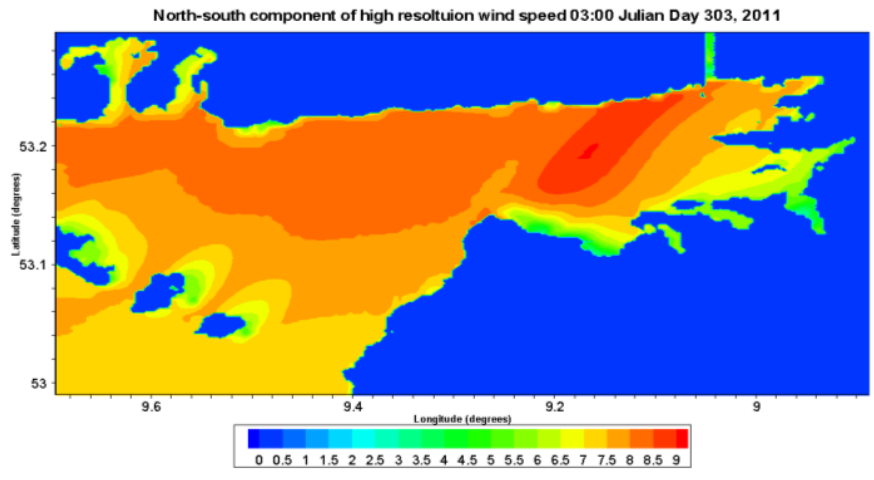

Fig. 8. Contour of north-south component of high resolution wind speed $(\mathrm{m} / \mathrm{s})$.

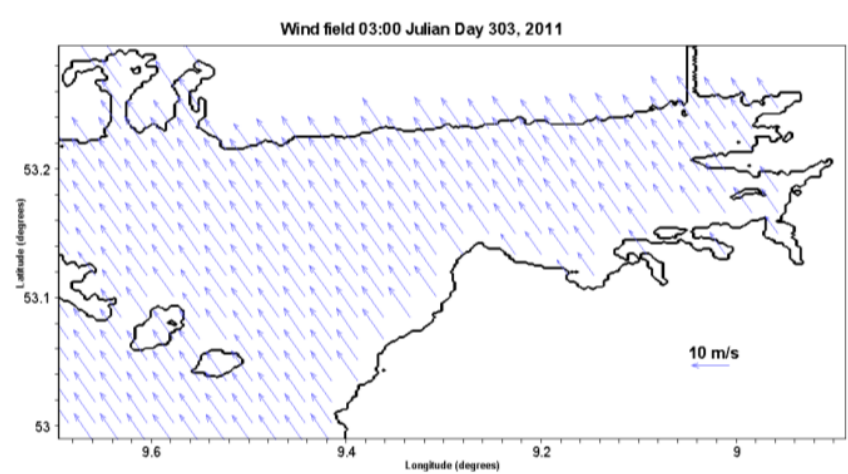

Fig. 9. Vector map of wind field from ARIMA.

\section{CONClusions}

In this research, two wind datasets were available. However, the high resolution wind data were not successive in time. High resolution wind fields were predicted based on the onshore IRUSE data and unsuccessive high resolution wind data because other parameters were not available for complete forecasting. Main conclusions of this research can be listed below:

1) Wind speed from onshore IRUSE station was always smaller than high resolution wind data from model HARMONIE in time.

2) Good agreement of wind direction existed between IRUSE wind data and HARMONIE high resolution wind data.

3) ARIMA prediction model with SPSS can be developed to forecast the high resolution wind fields.

4) Successive onshore wind data can be used to guide the forecasting of high resolution wind fields using available forecasted high resolution wind fields. 
The estimated high resolution wind fields will be used in numerical models in the future work.

\section{ACKNOWLEDGMENT}

L. Ren thanks Prof. M. Hartnett and Dr. S. Nash for the language improvements. L. Ren thanks D. Nagle for running the models and Dr. J. Sheahan for providing meteorological data. L. Ren thanks ICHEC (Ireland's High-Performance Computing Centre) for providing the computation platform.

\section{REFERENCE}

[1] M. H. Rio and F. Hernandez, "High-frequency response of wind-driven currents measured by drifting buoys and altimetry over the world ocean," Journal of Geophysical Research: Oceans, vol. 108, p. 3283, 2003.

[2] M.-H. Rio and F. Hernandez, "High-frequency response of wind-driven currents measured by drifting buoys and altimetry over the world ocean," Journal of Geophysical Research, vol. 108, p. 3283 , 2003.

[3] S. Kim, R. Samelson, and C. Snyder, "Ensemble-based estimates of the predictability of wind-driven coastal ocean flow over topography," Monthly Weather Review, vol. 137, pp. 2515-2537, 2009.

[4] J. Ding, "Impact of HARMONIE high-resolution meteorological forecasts on the air quality simulations of LOTOS-EUROS," Royal Netherlands Meteorological Institute, 2013.

[5] T. Perttula, P. Jokinen, and K. Eerola, "Impact of IASI in HARMONIE forecasting system during convective storm events in Finland during summer 2010," presented at the International TOVS Study Conference, Toulouse, France, 2012.

[6] S. L. Ho, M. Xie, and T. N. Goh, "A comparative study of neural network and Box-Jenkins ARIMA modeling in time series prediction,' Computers \& Industrial Engineering, vol. 42, pp. 371-375, 2002.

[7] J. L. M. Saboia, "Autoregressive Integrated Moving Average (ARIMA) models for birth forecasting," Journal of the American Statistical Association, vol. 72, pp. 264-270, 1977.

[8] K. Stanislav, D. Steinley, and L. Thombs, Statistics in the Social Sciences: Current Methodological Developments, 2010.

[9] P. Goyal, A. T. Chan, and N. Jaiswal, "Statistical models for the prediction of respirable suspended particulate matter in urban cities," Atmospheric Environment, vol. 40, pp. 2068-2077, 2006.

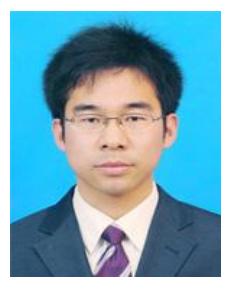

Lei Ren was born in Hubei province, China. He received the master degree in harbor, coastal and offshore engineering, College of Harbor, Coastal and Offshore Engineering, Hohai University in 2011. He is studying in water engineering as a $\mathrm{PhD}$ student in National University of Ireland, Galway from October, 2011 .

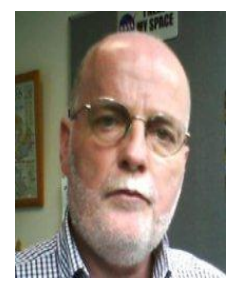

Michael Hartnett was born in Birmingham, England. $\mathrm{He}$ received his $\mathrm{Ph} . \mathrm{D}$. degree in civil engineering, Trinity College Dublin in 2000. He is a professor in the College of Engineering and Informatics, National University of Ireland, Galway. Prof. Michael Hartnett has published 40 journal papers and 63 conference papers. His scientific interest is marine modeling, tidal energy, water quality, climate change, coastal flooding, and data assimilation.

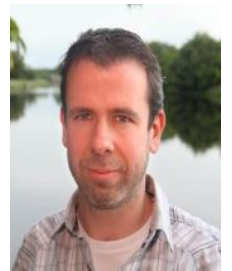

Stephen Nash received his Ph.D. degree in civil engineering, from National University of Ireland, Galway, in 2010. He is a lecturer in the College of Engineering and Informatics, National University of Ireland, Galway. Dr. Stephen Nash's scientific interest is physical and computer modeling of oceanic, estuarine and freshwater systems.

Jerome Sheahen is a lecturer in the School of Mathematics, Statistics and Applied Mathematics at National University of Ireland, Galway. His research interests include qualitative and quantitative robustness in linear models, multivariate analysis and time series, and finite and asymptotic distributions arising in applied probability.

Diarmuid Nagle was born in Ireland. He received the master's degree in civil engineering, National University of Ireland, Galway in 2013. His master's research involved the development of a three-dimensional hydrodynamic model of Galway Bay, Ireland. 